\title{
Effects of information and communication technology use in nursing and obstetric learning in low- and middle-income countries: A systematic review
}

\author{
Pilabré Arzouma Hermann*1, Ngangue Patrice ${ }^{1,2}$, Bationo Nestor ${ }^{1}$, Barro Abibata ${ }^{1}$, Pafadnam Yacouba $^{1}$, Soubeiga \\ Dieudonné ${ }^{1}$ \\ ${ }^{1}$ Institut de Formation et de Recherche Interdisciplinaires en Sciences de la Santé et de l'Éducation, IFRISSE \\ ${ }^{2}$ Département de médecine de Famille, Université MGill, Canada
}

Received: June 20, 2021

DOI: $10.5430 /$ jnep.v12n1p7

\author{
Accepted: August 16, 2021 \\ Online Published: August 27, 2021
}

\begin{abstract}
Introduction and objective: Studies on the effects of information and communication technology (ICT) use in nursing and obstetric learning in low- and middle-income countries are limited despite growing scientific evidence that online learning has positive effects. This systematic review aims to identify and synthesize the effects of information and communication technologies utilization in nursing and obstetric learning in low- and middle-income countries.

Methods: A search of articles published from 2016 to 2020 on the effects of ICT use in nursing and obstetric learning was conducted in PubMed, CINAHL, Epistemonikos and ERIC.

Results: Of 483 articles identified, eleven were reviewed, and eight were found to be relevant. The included articles were synthesized into a narrative synthesis. The effects of using ICT in learning are related to student motivation, autonomy in learning, meaningful acquisition of knowledge and skills. Furthermore, students have a positive perception of the use of ICT in learning. Conclusions: The results of this study on the use of ICTs in nursing and obstetric sciences learning in low- and middle-income countries show that ICTs are used primarily as a medium for distance learning. In addition, it was found that e-learning has several advantages or positive effects. However, many students do not have a personal computer, and they have low or average skills in the use of computer tools, and access to the Internet is low. A limitation of this study is the lack of primary data on the effects of ICT use in obstetric sciences learning in low- and middle-income countries.
\end{abstract}

Key Words: Information and communication technologies, E-learning, Systematic review, Nursing and obstetrics, Low and middle-income countries

\section{INTRODUCTION}

The rapid evolution of information and communication technologies (ICT) has led to the development of applications used in everyday life and all sectors of activity. ${ }^{[1]}$ In facing this development, the integration of ICT has been imposed on educational systems. ${ }^{[2]}$ The use of ICT in learning has become commonplace in higher education and, consequently, in nurses, midwives, and other health professionals worldwide. ${ }^{[3]}$ The benefits of ICT in education are well known. ICT improves the quality of teaching and learning in several ways. It facilitates the acquisition and appropriation of knowledge through better access to educational resources,

\footnotetext{
* Correspondence: Pilabré Arzouma Hermann; Email: hpilabr@yahoo.com; Address: Institut de Formation et de Recherche Interdisciplinaires en Sciences de la Santé et de l'Éducation, IFRISSE.
} 
enrichment of these resources, stimulating pedagogical relationships, and greater involvement of learners in the learning process. ${ }^{[4]}$ Automatically, the integration of ICT in teaching induces students to use ICT in their learning. Currently, ICT tools are used as a support in online teaching/learning. As a result, the use of ICT in teaching/learning is often reduced to online distance learning by some users. There are different models or designs of e-learning that are used in practice. The most common are: the enhanced model, the blended e-learning model, and the fully online model. The latter model can be divided into two categories: synchronous and asynchronous. ${ }^{[5]}$

In response to the COVID-19 pandemic, many governments worldwide decided to close educational institutions to contain the disease. ${ }^{[6]}$ Colleges and universities were forced to convert all face-to-face courses to an online learning format. ${ }^{[6]}$ Indeed, most universities have moved to online learning in a synchronous and/or asynchronous environment. ${ }^{[6]}$ E-learning has been defined as an educational method that facilitates learning by applying information and communication technologies, offering learners access to all required educational programs. ${ }^{[5]}$

Studies and systematic reviews have been conducted to identify the effects of using e-learning in teaching/learning in health sciences and specifically in nursing. However, to our knowledge, few systematic reviews have been conducted to identify the effects of ICT use, as a whole, in nursing and obstetric sciences learning in low and middle income countries.

In the context of the West African Health Organization (WAHO) member countries, which are all low and middleincome countries, nursing and obstetrics students are trained in the same school. Better yet, they take standard core courses in the first and second semesters of their studies. ${ }^{[7]}$

A systematic review type of research deserves to be done to identify the effects of using ICT in learning, especially in the current context where the use of ICT in higher education and training institutions is widespread and intensifying since the advent of the COVID-19 pandemic. ${ }^{[8]}$

This review will focus on the effects of ICT use in the learning of nursing and obstetric sciences students in low and middle income countries. The question of the systematic review is: what are the effects of ICT use in nursing and obstetric sciences learning in low- and middle-income countries?

The objectives of this study are to (1) identify patterns of ICT use and (2) synthesize the effects of ICT use in the learning of nursing and obstetric sciences students in low- and middle-income countries. The effects of ICT use can be defined as the intended and unintended changes that are directly or indirectly attributable to the integration of ICT into teaching-learning.

The results of this systematic review provide educational institutions in low- and middle-income countries with evidence of the effects of using ICTs in nursing and obstetrical education and the conditions to achieve these effects.

\section{Methods}

The methodology followed the Preferred Reporting Items for Systematic Review and Meta-Analysis Protocols (PRISMAP). ${ }^{\text {[9] }}$ Due to the diversity of methods used in the studies, a narrative synthesis was performed. The systematic review protocol was registered at the International prospective register of systematic reviews under the number CRD42020219619.

\subsection{Eligibility criteria}

All original research articles on the effects of ICT use meeting the following eligibility criteria were included:

1) research with a quantitative, qualitative, or mixed design;

2) studies with nursing and/or obstetrical students and faculty as participants;

3) the use of ICT in nursing and/or obstetric sciences learning in low- and middle-income countries;

4) articles published in English or French journals from 2016 to 2020 .

Exclusion criteria were:

1) articles on the use of ICT in learning whose participants are pre-licensure nursing and/or obstetrics students;

2) commentaries, review papers, case studies, letters, discussion papers, posters, conference abstracts, conference reports, and dissertations;

3) full text not available.

\subsection{Information sources}

Literature search strategies were developed based on free and controlled vocabularies. We searched PubMed, CINAHL, Epistemonikos and ERIC databases. The search was limited to English or French language and human subjects. To ensure saturation of the literature, additional searches were conducted in the bibliography of articles from the search. Hand searches were also conducted in academic journals to identify additional relevant studies.

\subsection{Search Strategies}

The search strategy for the PudMed database was as follows: ("Students" [MeSH] OR "Learners" OR "Teachers" OR "Trainers" OR "Campus managers" OR "Directors" OR 
"Education, Graduate" [MeSH] OR "Universities" [MeSH] OR "Faculty" [MeSH] OR "Africa" [MeSH]) AND ("Information and communication technologies for education" OR "ICT for education" OR "Web-based learning" OR "Elearning" OR "Distance education" OR "Distance Learning" [MeSH] OR "Learning, Distance" [MeSH] OR "Computerized technological resources" OR "Online Learning" [MeSH] OR "Learning, Online" [MeSH] OR "Online Education" [MeSH] OR "Remote Education" OR "Remote instruction" OR "Virtual classes" OR "Virtual classroom" OR "Integration of ICT" OR "ICT" OR "Internet use" [MeSH] OR "Computer User Training" [MeSH]) AND ("Access to ICT" OR "Use of ICT" OR "Capacity of use" OR "Perceived usefulness" OR "Confirmation of expectations" OR "Student satisfaction" OR "Health knowledge, attitudes, practice" OR "Health Knowledge, Attitudes, Practice" [MeSH] OR "Student engagement" OR "Academic Success" [MeSH] OR "Learning" OR "Professional Competence" [MeSH] OR "mental competency" [MeSH] OR "Skills").

This strategy was adapted for use in the electronic bibliographic databases Epistemonikos, ERIC, and CINAHL (Appendices). The last search on these databases was performed on October 30, 2020.

\subsection{Recording of studies}

Articles identified during database searches were exported into Zotero software. Data were extracted using a form designed on Microsoft Excel. The data were extracted and tabulated by one author of the manuscript and verified by another author. Disagreements were resolved by discussion between the two authors.

\subsection{Data elements}

We extracted data on the first author, country of origin, year of study publication, study scope, design, participants, study duration, data collection, methods of analyses, intervention, control, and primary outcomes. Some of these extracted data were presented in tabular form in the Results section.

\subsection{Results and prioritization}

The main outcomes are the effects of using ICT in learning, which can be defined as the expected or unexpected changes attributable directly or indirectly to the use of ICT in learning. These effects were: student motivation, autonomy in learning, satisfaction, and acquisition of knowledge and skills.

Secondary outcomes are those elements that contribute to the production of effects. These elements were: access to ICT, ability to use ICT, perceived usefulness of ICT and confirmation of expectations.

\subsection{Risk of bias in individual studies}

The methodological quality of the eight studies included in this synthesis was assessed using the Mixed Methods Appraisal Tool (MMAT). The MMAT is a critical appraisal tool designed for mixed systematic reviews, i.e., reviews that include studies using qualitative, quantitative, and mixed methods. It assesses the methodological quality of five categories of studies: qualitative research, randomized trials, quantitative descriptive studies, and mixed-methods studies. ${ }^{[10]}$ The MMART criteria list includes two triage questions and five questions per study category. In addition, the document includes indicators that explain and illustrate some criteria. For each question, the authors responded by checking "Yes", "Don't know", or "No". One author evaluated six articles, and another evaluated two articles. The authors discussed the results of the assessment for all included articles with particular attention to questions that were checked "Don't know" or "No." In terms of the methodological quality of the articles, one was of low quality, three of medium quality, and four of good quality.

\subsection{Data synthesis}

The main findings of the studies were analyzed and summarized in a narrative manner. Indeed, a systematic narrative synthesis was provided with the information presented in the text and tables to summarize and explain the characteristics and results of the included studies.

\section{Results}

\subsection{Search results}

The flow and number of studies examined at each stage of this systematic review are presented in Figure 1. A total of 486 articles were retrieved through various searches. After removing duplicates, 436 remained, and eight met the inclusion and exclusion criteria.

\subsubsection{Characteristics of the studies}

The included articles were published in English between 2016 and 2020. One study was conducted in a low-income country, ${ }^{[3]}$ and seven studies were conducted in four middleincome countries. ${ }^{[1-17]}$ Five studies focused on teaching and learning of specific subjects such as ethics and deontology ${ }^{[11]}$ research course, ${ }^{[17]}$ clinical ear examination, ${ }^{[12]}$ health information management, gerontology nursing, ${ }^{[13]}$ international classification of nursing practice. ${ }^{[15]}$ The other three studies evaluated, in a global way, the effects of the use of ICT, more particularly e-learning in learning and the realization of certain pedagogical activities such as learning assessment. ${ }^{[3,14,17]}$ 


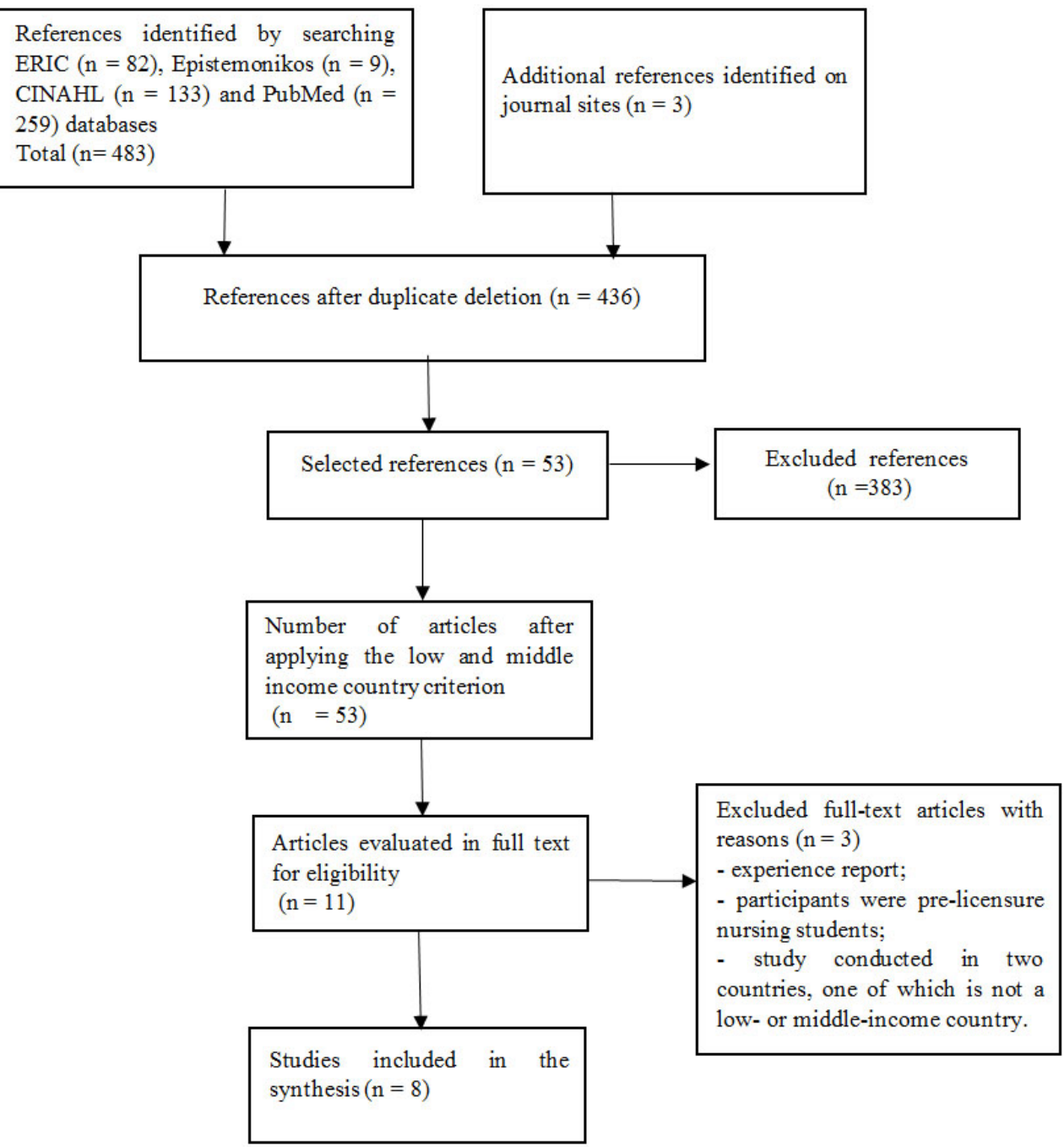

Figure 1. Adapted PRISMA flow chart

The modes of intervention examined by the eight studies involved synchronous and/or asynchronous online learning. Of the eight studies, four used quantitative methods, ${ }^{[11,13,14,17]}$ one used qualitative methods ${ }^{[12]}$ and three used mixed methods. $^{[3,15,16]}$ Almost all studies specified the methodological approach used. Of the eight included studies, two used the quasi-experimental approach, ${ }^{[11,13]}$ two used the descriptive approach ${ }^{[14,17]}$ two used the sequential mixed approach, ${ }^{[12,16]}$ one used the qualitative descriptive approach, and one used the convergent mixed approach. ${ }^{[3]}$

Seven included studies focused exclusively on nursing students as participants. ${ }^{[11-14,17]}$ One study included faculty, information and communication technology managers, and campus managers. ${ }^{[3]}$

\subsubsection{Individual results of the included studies}

The individual results of the included studies are shown in Table 1.

\subsection{Narrative synthesis}

\subsubsection{General informations}

- How are information and communication technologies used in learning?

Information and communication technologies (ICTs) are used in various ways in teaching/learning generally and in nursing teaching/learning specifically. All eight articles included in this systematic review address the use of information and communication technologies as a medium for distance or online teaching/learning. ${ }^{[3,11-17]}$ These articles describe different models of online learning. Four articles describe the blended e-learning modality, i.e., integrating faceto-face learning experiences with online learning. ${ }^{[11,12,15,16]}$ One article described the blended online learning model using a smartphone. ${ }^{[15]}$ One article described the e-learning model in a synchronized and asynchronized environment, and another article described the e-learning model in an asynchronized environment. ${ }^{[17]}$ Two articles discussed online 
learning without further details. ${ }^{[3,14]}$

\section{- Access to ICT}

Access to a computer and connection is the first requirement for using information and communication technology in learning. ${ }^{[3]}$ In terms of owning a desktop or laptop computer, most nursing students reported not owning a personal desktop or laptop computer. ${ }^{[14]}$ However, most students report using a desktop or laptop computer and the Internet for learning. ${ }^{[12]}$ In terms of Internet access, slightly more than half of the students accessed the Internet 1 to 3 times per day. ${ }^{[12]}$ In addition, about half of the students spent $1-3$ hours on the Internet per day. ${ }^{[12]}$

\section{- Capacity to use ICT}

Most students have poor or lacking skills in using the Internet. ${ }^{[13]}$ Some have moderate or no computer skills. ${ }^{[12]}$ This could be because many nursing students do not receive formal computer training. Therefore, many cannot use computer application software very well. ${ }^{[14]}$

\subsubsection{Effects of the use of ICT in learning}

\section{- Student motivation}

The use of ICT in teaching and learning motivates nursing students. ${ }^{[13]}$ This motivation is reflected in an improved classroom climate, increased student focus on learning, etc. ${ }^{[3]}$ Several factors could explain students' motivation to use ICT in their learning. Indeed, students find that they save time in travel, especially when they have to attend classes regularly. In addition, students indicate that they can receive messages from their peers and teachers without necessarily being at school. They also mention that the use of ICT in learning facilitates collaborative work among students. ${ }^{[3]}$

\section{- Autonomy in learning}

The use of ICT in learning promotes autonomy in learning. Indeed, the use of ICT allows students to take courses at any time without necessarily being at school. In addition, students have access to learning resources anywhere and anytime. ${ }^{[3]}$ However, few students had access to online medical journals or other online learning resources. ${ }^{[12]}$

- Meaningful acquisition of knowledge and skills

The use of ICT in learning improves the acquisition of knowledge and skills. It promotes the improvement of students' performance and professional skills. ${ }^{[14]}$ Indeed, the use of ICT promotes a clear understanding of the course, ${ }^{[15]}$ makes it easy to understand the learning object, ${ }^{[11]}$ and the level of knowledge acquisition high. ${ }^{[16]}$ Moreover, the forum resource allows, even at a distance, an interaction between students favouring a collective construction of knowledge. ${ }^{[16]}$

- Perception of usefulness

Nursing students have a positive perception of the outcomes

Published by Sciedu Press of using online learning. Respondents' perceptions of the outcomes of online learning were broken down into 12 items, which are: online peer support was helpful; the course project is in line with their expectations; they gained more knowledge about technology; they gained skills in using the Internet; developed new ICT skills, changed their attitude; be able to use the new skill throughout their career; applied the new knowledge in their life; initiated new ideas from the new knowledge; interactive exchanges were essential in the course. ${ }^{[3]}$ In addition, students have a sense of confidence in using different forms of technology. ${ }^{[17]}$ However, some students, mainly undergraduates, find the distance modality still a new experience, which causes some apprehension. ${ }^{[16]}$ Teachers believe that online learning improves the quality of memorization. Indeed, students easily remember what they have learned. ${ }^{[3]}$

\section{Discussion}

This study systematically examined the effects of ICT use in nursing and obstetrics learning. The results indicate that ICT are used as a medium for online learning. ${ }^{[3,11-17]}$ Most students do not have a personal desktop or laptop computer. However, for online learning, they use desktop or laptop computers with an Internet connection. The articles included in this study show that most students have low skills or lack of skills in using the Internet. ${ }^{[13]}$ Some have average or moderate computer skills. ${ }^{[12]}$

On the other hand, studies have reported that using ICT has advantages or beneficial effects in learning nursing and obstetrics. Indeed, the use of ICT in teaching/learning makes students motivated, promotes autonomy in learning, and improves the acquisition of knowledge and skills. ${ }^{[3,13,14]}$ In addition, nursing students have positive perceptions of the outcomes of using e-learning. ${ }^{[3]}$ No study has presented a theoretical framework used to assess the effects of ICT use in learning.

Most students reported not owning a personal desktop or laptop computer. ${ }^{[14]}$ However, the majority of students use a desktop or laptop computer and the Internet. ${ }^{[12]}$ This result implies that these students do not use smartphones but use the laptops or desktops of their relatives for their online learning. Similar to this review, a previous review reported that ICT access, technical issues, and internal support are concerns for both students and faculty. ${ }^{[5]}$ The problem of ICT accessibility could be explained by the lack of appropriate institutional strategies to promote ICT use. ${ }^{[5]}$ It could also be explained by the unfavourable socio-economic situation of the students' parents. ${ }^{[18]}$ This lack of access to ICT negatively affects its use in learning, as the computer and the Internet connection are the primary resource for online learning. ${ }^{[3]}$ 
Most students have poor or lacking skills in using the Internet. ${ }^{[13]}$ Some have average or no computer skills. ${ }^{[12]}$ These results must be considered because they were obtained based on self-reporting and not based on an assessment. A previous systematic review highlights a lack of technical skills among students as a problem related to ICT use in teaching/learning. ${ }^{[5]}$ The results of another systematic review indicate that a lack of computer skills was identified as a major barrier to students using computer-based learning methods, rather than a lack of preference for new technologies. ${ }^{[2]}$ Students' inadequate preparation explains the lack of computer and Internet skills. Students rarely receive any significant computer and Internet use training before moving on to use ICT in their learning. ${ }^{[5]}$ This lack of skills leads to the loss of resources and demotivation of students to use ICT in their learning. ${ }^{[5]}$

The use of ICT in teaching/learning motivates students. ${ }^{[13]}$ This motivation results in an improved classroom climate increased student focus on learning. ${ }^{[3]}$ Webb et al. found significantly higher engagement online than in the classroom. ${ }^{[5]}$ Improved student engagement was a feature of online learning. ${ }^{5]}$ However, studies reviewed in a systematic review report problems with student motivation and a general lack of educational technology enthusiasm. ${ }^{[5]}$ This difference could be explained by how universities prepare their students for e-learning, the accessibility of ICT, and the educational levels of the participants in the study. Indeed, studies have shown that undergraduate students are apprehensive about using ICT in learning. ${ }^{[15]}$ Therefore, the effectiveness of student learning depends on the timing and quality of student preparation for online learning.

The results of this systematic review show that the use of ICT in learning promotes autonomy in learning. Indeed, the use of ICT allows students to take courses at any time without necessarily being at school. Many studies have found advantages to asynchronous learning, particularly time-saving and self-paced learning. ${ }^{[5]}$ This advantage could be related to the fact that students have access to resources at any time and place for their learning. If the conditions are met, the adult learner can initiate initiative, autonomy, and engagement in the learning process. This suggests that autonomy in online learning requires well-designed and accessible learning resources. Hence, it is essential to have quality equipment and train teachers and students accordingly to use ICT in teaching and learning. The development of autonomy allows students to become aware of their responsibility in the learning process. This will increase their motivation. Motivation is a prerequisite for successful learning. ${ }^{[19]}$

The use of ICT in learning improves the acquisition of knowl- edge and skills. It enhances students' performance and professional skills. ${ }^{[14]}$ Previous studies have found similar results, revealing a significant improvement in clinical skills or knowledge of students accessing online material. ${ }^{[5]}$ This improvement in knowledge or skill acquisition is thought to be because students can use the learning resources as many times as they wish to understand the course better. These effects are often observed in blended learning, where there is an integration of face-to-face learning experiences with online learning. ${ }^{[5]}$

Moreover, in online learning, the forum resource allows, even at a distance, an interaction between students favouring a collective construction of knowledge. ${ }^{[1]}$ Indeed, adult learning is easy in an atmosphere that promotes collaboration through participation. In addition, collaborative online learning has eight advantages that have been identified by a study. ${ }^{[20]}$ However, to achieve beneficial effects of online learning, an emphasis must be placed on the availability of infrastructure and equipment, the availability of human resources, and teachers and students training. The improvement of students' performance through e-learning depends on their motivation and ability to use ICT tools. ${ }^{[21]}$

Nursing students have a positive perception of the outcomes of using online learning. This positive perception is expressed through positive attitudes. A previous systematic review reports that many of the studies reviewed found positive student attitudes toward online and electronic learning media. ${ }^{[5]}$ This positive perception is thought to be due to the many benefits that online learning offers. This suggests that students are willing to do their learning online. However, studies report that the majority of students do not want exclusively online learning. ${ }^{[13]}$ This student desire is evidence that blended online learning is the best model for using ICT in teaching/learning. Blended learning has several advantages: the successful fusion of face-to-face and online aspects by making resources more accessible. It promotes the student-centred approach, provides various materials, increases participation, and promotes student-student/teacherstudent interaction. In addition, it allows for timely feedback and creates a ground for synchronous and asynchronous discussions. ${ }^{[13]}$

\section{Study limitations}

The systematic review includes a sample of eight articles because a limited number of articles met the criteria. The small number of relevant articles included in this systematic review suggests a conservative interpretation of the results. In addition, we initially chose to include articles in which the participants were nursing and obstetrics students. We were unable to find articles with obstetrical students as par- 
ticipants. Therefore, applying the results of this study to obstetrical science students must be done with caution.

\section{Conclusion}

The results of this systematic review indicate that ICT is used primarily as a support for online learning. These results support the findings of other studies on the effects or benefits of ICT use in general and nursing learning in particular. However, this review shows that many students do not have personal computers, access to the Internet is low, and most students have low to moderate skills in using computer tools. In addition, this study highlighted the lack of primary data on the effects of ICT use in obstetric science learning in lowand middle-income countries. We suggest that research be conducted on the effects of ICT use in obstetric sciences learning in low- and middle-income countries.

\section{ACKNOWLEDGements}

The authors would like to thank Dr Dieudonné Soubeiga for initiating the systematic review training and Dr Emile Pargui Paré for agreeing to financially support the students.

\section{CONFLicts OF INTEREST Disclosure}

The authors declare that there is no conflict of interest.

\section{REFERENCES}

[1] Poyet F. Comprendre la construction des usages des TIC en formation: vers un modèle d'analyse systémique. Université Claude Bernard Lyon 1; 2014

[2] Riyami B. Analyse des effets des TIC sur l'enseignement supérieur au Maroc dans un contexte de formation en collaboration avec une université française. 2018; 168.

[3] Harerimana A, Mtshali NG, Hewing H, et al. E-Learning in Nursing Education in Rwanda: Benefits and Challenges. An Exploration of Participants' Perceptives. 2016. https://www.researchgate.n et/publication/299598225

[4] Ben Youssef A, Rallet A, Usage des TIC. dans l'enseignement supérieur. Réseaux Découv [Internet]. 2009; 155(3): 9. https: //doi.org/10.3917/res.155.0009

[5] Regmi K, Jones L. A systematic review of the factors-enablers and barriers-affecting e-learning in health sciences education. BMC Med Educ. 2020; 20(1): 91. PMid:32228560 https://doi .org/10.1 186/s12909-020-02007-6

[6] Pilabré AH, Ngangue P, Barro A, et al. An Imperative for the National Public Health School in Burkina Faso to Promote the Use of Information and Communication Technologies in Education During the COVID-19 Pandemic: Critical Analysis. JMIR Med Educ. 2021; 7(2): e27169. PMid:33970868 https://doi.org/10.2196/27169

[7] Organisation ouest africaine de la santé. Curriculum de fomation harmonisé des infirliers et sages-femmes des pays membres de la CEDEAO. 2010

[8] Ibrahim G, Luzinge H, Kapanda G. Teaching and Learning Experiences in Medical Education During the COVID-19 Pandemic: The Case of Kilimanjaro Christian Medical University College (KCMUCo), Tanzania. J Learn Dev. 2020; 7(3): 433-46.

[9] Shamseer L, Moher D, Clarke M, et al. Preferred reporting items for systematic review and meta-analysis protocols (PRISMA-P) 2015: elaboration and explanation. BMJ. 2015; 349(jan02 1): g7647-g7647. PMid:25555855 https://doi.org/10.1136/bmj.g7647

[10] Hong QN, Pluye P, Fàbregues S, et al. Mixed methods appraisal tool (MMAT) version française. 2018.

[11] Chao SY, Chang YC, Yang SC, et al. Development, implementation, and effects of an integrated web-based teaching model in a nursing ethics course. Nurse Educ Today. 2017; 55: 31-7. PMid:28511085 https://doi.org/10.1016/j.nedt.2017.04.011

[12] Willemse JJ, Jooste K, Bozalek V. Experiences of undergraduate nursing students on an authentic mobile learning enactment at a higher education institution in South Africa. Nurse Educ Today. 2019; 4: 6975. PMid:30594902 https://doi.org/10.1016/j.nedt. 2018. 11.021

[13] Ali KAG, Khalil HEM, El-Sharkawy FM. Impact of Online Remote Education on the Learning Process among Nursing Students. Open J Nurs. 2020; 10(09): 810-30. https://doi.org/10.4236/ojn. 2020.109057

[14] Irinoye O, Ayamolowo S, Tijnai OK. Part-Time Undergraduate Nursing Students' Perception and Attitude to ICT Supports for Distance Education in Nursing in Nigeria. 2016; 4(2): 14.

[15] Avelino CCV, Costa LCS da, Buchhorn SMM, et al. Teachinglearning evaluation on the ICNP $($ using virtual learning environment. Rev Bras Enferm. juin 2017; 70(3): 602-9. PMid:28562810 https://doi.org/10.1590/0034-7167-2016-0545

[16] Luo S, Yang HH. Using Technologies in Nursing Research Education: A Mixed Methods Case Study. CIN Comput Inform Nurs. 2018; 36(6): 293-304. PMid:29494359 https ://doi . org/10.1097/CI N. 0000000000000420

[17] Bello UL, Elshafie IkF, Yunusa U, et al. Utilization of Information and Communication Technology among Undergraduate Nursing Students in Tanta University, Egypt. Nurs Prim Care. 2017; 1(6): 1-8. https://doi.org/10.33425/2639-9474.1038

[18] Karsenti T (dir). Intégration pédagogique des TIC en Afrique: Stratégies d'actions et piste de réflexion. CRDI Ottawa; 2009.

[19] Zhuya W. Une pédagogie mettant l'accent sur l'autonomie de l'apprenant. 2011; 9.

[20] Castillo-Merino D, Serradell-López E, Vilaseca-Requena J. Usage des technologies de l'information et de la communication dans l'enseignement supérieur: Une analyse des performances des étudiants en e-learning dans la région catalane. Réseaux. 2009; 155(3): 55. https://doi.org/10.3917/res.155.0055

[21] Walckiers M, De Praetere T. L'apprentissage collaboratif en ligne, huit avantages qui en font un must. Distances et savoirs. 2004; 2(1): 53-75. https://doi.org/10.3166/ds.2.53-75 